\title{
NOUVELle
}

\section{Nouvelles perspectives pour prévenir le travail préterme}

Mathieu Nadeau-Vallée ${ }^{1}$, Julia Palacios ${ }^{2}$, Amarilys Boudreault ${ }^{2}$, Sylvain Chemtob ${ }^{1}$
${ }^{1}$ Département de pédiatrie, pharmacologie et ophtalmologie, CHU Sainte-Justine, centre de recherche,

3175 chemin Côte-Sainte-Catherine, Montréal, H3T 1 C5 Canada ;

${ }^{2}$ Département de médecine, université de Montréal, 2900 boulevard Édouard-Montpetit, Montréal, H3C 3J7 Canada.

sylvain.chemtob@umontreal.ca mathieu.nadeau-vallee@umontreal.ca
> La prématurité est un facteur de risque dans plus de $50 \%$ des complications néonatales, dont la sévérité augmente avec la diminution de l'âge gestationnel. Les naissances prématurées concernent plus de $10 \%$ des naissances dans le monde $[1,11]$. De plus, il n'y a pas de grande disparité entre l'incidence des naissances prématurées dans les pays en développement et celle qui est observée en Amérique du Nord, démontrant que les progrès faits en médecine à cet égard n'ont pas été concluants. Quatre éléments l'expliquent en grande partie : (1) les agents pharmacologiques utilisés pour arrêter le travail (les tocolytiques ${ }^{1}$ ) sont administrés aux femmes symptomatiques déjà en travail, et ne prolongent généralement la durée de gestation que de 48 h au plus; (2) l'administration préventive d'agents tocolytiques impliquerait d'identifier précocement les femmes à risque, et les tests mis au point dans ce but n'ont que de faibles valeurs prédictives; (3) les tocolytiques sont des agents non sélectifs induisant des effets secondaires qui limitent leur usage prophylactique; (4) les tests de dépistage ne sont pas utilisés en routine chez les femmes primé-gravides (première gestation) ou multi-gravides (plus d'une gestation) à faible risque [2]. Bien que les taux de mortalité associés à la prématurité soient en baisse ces dernières années en raison de l'amélioration des

\footnotetext{
${ }^{1}$ Le traitement vise à arrêter ou diminuer les contractions utérines. La force et la fréquences des contractions spontanées sont controlées par les canaux calciques et potassiques, des neurotransmetteurs (système nerveux autonome), certaines hormones et métabolites locaux (ocytocine, prostaglandines, progestérone, catécholamines, etc.).
}

soins postnatals, celle-ci demeure un grave problème en raison de la morbidité importante, et représente un coût social et économique énorme.

Parmi les tocolytiques approuvés et utilisés en clinique (sympathicomimétiques, magnésium, anti-inflammatoires non stéroidiens, antagonistes du récepteur de l'ocytocine, bloqueurs de canaux calciques, etc.), aucun ne s'est avéré plus efficace que le placebo pour améliorer les complications néonatales engendrées par la prématurité. La justification essentielle d'une administration de tocolytiques est qu'elle prolonge la période in utero suffisamment pour permettre l'administration de corticostéroïdes. Ceux-ci accélèrent la maturation pulmonaire fœtale et diminuent la fréquence de la maladie des membranes hyalines et la mortalité périnatale chez les nouveau-nés prématurés [12].

$\varepsilon$ n revanche, l'utilisation de tocolytiques est associée à des effets secondaires en raison de leur mode d'action non sélectif. On en distingue plusieurs : (1) les sympathicomimétiques ont des effets secondaires majeurs, surtout s'ils sont utilisés en combinaison avec les corticostéroïdes, puisqu'ils agissent sur les récepteurs adrénergiques d'expression ubiquitaire ; (2) le magnésium fait obstacle au calcium (qui est requis pour la contraction myométriale) de manière non spécifique, et il induit des effets secondaires musculaires; (3) les anti-inflammatoires non stéroïdiens agissent en inhibant l'enzyme prostaglandine-endoperoxide synthase 2 ( $C O X-2)$ de manière non spécifique, créant chez le fœtus des effets rénaux et cardiaques non désirés ;
(4) les antagonistes du récepteur de l'ocytocine pourraient agir également sur le récepteur de la vasopressine, entraînant hypotension et palpitations cardiaques; (5) les inhibiteurs des canaux calciques provoquent une augmentation de la pression artérielle et des risques d'angor [2, 3]. On comprend donc qu'une administration de ces agents ne puisse pas constituer une thérapie préventive chez les femmes à risque d'accouchement prématuré. Elle est plutôt indiquée chez les femmes symptomatiques en phase de travail préterme, mais leur efficacité dans ce contexte est faible.

Notre laboratoire - en concertation avec d'autres laboratoires qui identifient des biomarqueurs prédictifs d'un déclenchement prématuré du travail [4] - a pour objectif de développer une approche dépourvue d'effets secondaires majeurs; elle devra donc être très sélective et cibler les mécanismes d'activation précoce de l'utérus pour diminuer les probabilités d'un travail précoce.

\section{L'induction du travail utérin}

L'activation de l'utérus est la conséquence de l'inflammation. Il est maintenant globalement accepté que le déclenchement précoce de la cascade inflammatoire menant à l'induction du travail pourrait expliquer les naissances prématurées [5]. Dans ces situations, un stimulus inflammatoire (infection, stress, etc.) provoque l'activation de l'immunité innée dans les tissus reproducteurs maternels. Les cellules de la réponse immunitaire innée (macrophages, cellules dendritiques, etc.) s'activent et sécrètent des cytokines pro-inflamma- 
toires et des chimiokines qui provoquent l'induction d'une cascade inflammatoire locale et la production des protéines activatrices de l'utérus (UAP; uterine activation proteins) [6]. Ces protéines comprennent des métalloprotéinases matricielles (MMP : matrix metalloproteinases), les récepteurs des agents ocytociques (OXTR : oxytocin receptor, FP : prostaglandin $F_{2 \alpha}$ receptor) ainsi que la connexine-43 (CX-43). Les MMP sont impliquées dans le remodelage cervical qui permet la dilatation du col de l'utérus, et interviennent aussi dans la fragilisation des membranes fœtales. OXTR et FP répondent à l'ocytocine et à la prostaglandine $F_{2 \alpha}$ respectivement, et déclenchent la contraction via une mobilisation calcique. Finalement, CX-43 est une protéine constituante des jonctions de type GAP qui favorisent une contractilité uniforme des cellules musculaires lisses. La cascade inflammatoire menant à l'induction des UAP et au commencement du travail est résumée dans la Figure 1. L'induction des UAP est favorisée par les cytokines, notamment les interleukines (IL)-1 et -6. Cibler les cytokines pro-inflammatoires, ou encore des médiateurs majeurs de l'inflammation dont NF- $\kappa B$ et les prostaglandines, pourrait permettre d'interrompre cette activation prématurée de l'utérus. Toutefois, cette approche demeure risquée pour deux raisons: (1) bien que la réaction inflammatoire joue un rôle dans la pathophysiologie du travail préterme, elle participe aussi au travail utérin lors de l'accouchement à terme ; par exemple, il a été démontré que l'inhibition de NF- $\kappa B$ serait nuisible à la cytoprotection [7] ; (2) l'immunosuppression engendrée par un agent anti-inflammatoire trop puissant peut entraîner une susceptibilité aux infections, qui serait délétère pour la mère et le fœtus. II est donc peu probable que ces anti-NF- $\kappa B$ soient un jour approuvés dans cette indication chez la femme enceinte.

\section{Molécules inhibant l'activité} des récepteurs de cytokines

Les molécules bloquant l'activation des récepteurs de cytokines disponibles sur le marché ont toutes un mode d'action compétitif, c'est-à-dire qu'elles se lient au site orthostérique du récepteur, ou empêchent complètement la liaison du ligand naturel. Cela provoque une inhibition complète de toutes les voies de signalisation du récepteur, qui est responsable des deux problèmes cités précédemment limitant leur usage en obstétrique. Une possibilité serait de développer un agent anti-inflammatoire ayant une sélectivité pharmacologique. C'est le cas des agents allostériques qui se lient sur un site autre que le site orthostérique, et modulent l'activité du récepteur sans la bloquer complètement. Ces agents sont beaucoup plus sélectifs que les agents compétitifs puisqu'ils ciblent certaines voies de signalisation d'un récepteur plutôt que d'inhiber toutes les voies; les effets indésirables en seraient réduits [8]. Un agent modulateur de récepteur de cytokine pourrait ainsi permettre d'atténuer le signal des cytokines et diminuer l'activation prématurée de l'utérus sans induire d'immunosuppression et sans nuire à l'accouchement normal à terme. Un peptide non compétitif bloquant le récepteur de la prostaglandine $\mathrm{F} 2 \alpha$ a d'ailleurs été récemment caractérisé et s'est révélé très efficace pour prévenir le travail préterme induit par l'inflammation chez la souris [9] ; l'efficacité a été confirmée dans des études cliniques de phase lb chez l'homme [10].

$\varepsilon$ n résumé, les tocolytiques actuellement disponibles en clinique sont inefficaces avant tout parce qu'ils sont administrés trop tard, lorsque le travail a déjà commencé. Leur mode d'action non spécifique est associé à de multiples effets secondaires chez la mère et le fœtus, ce qui limite grandement leur usage

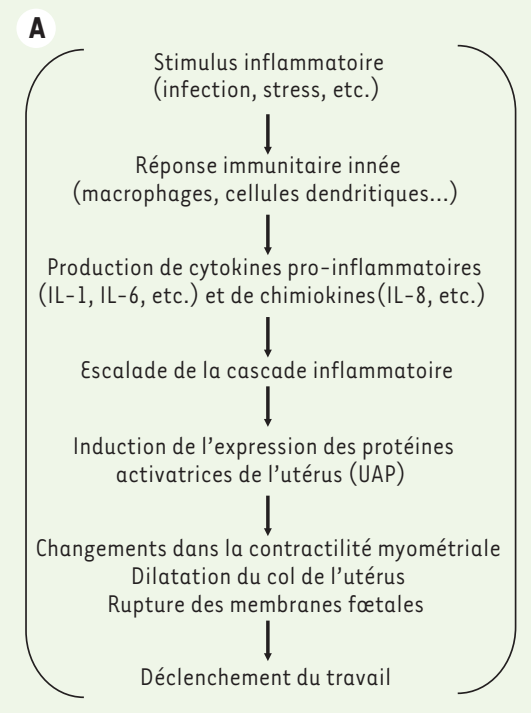

B

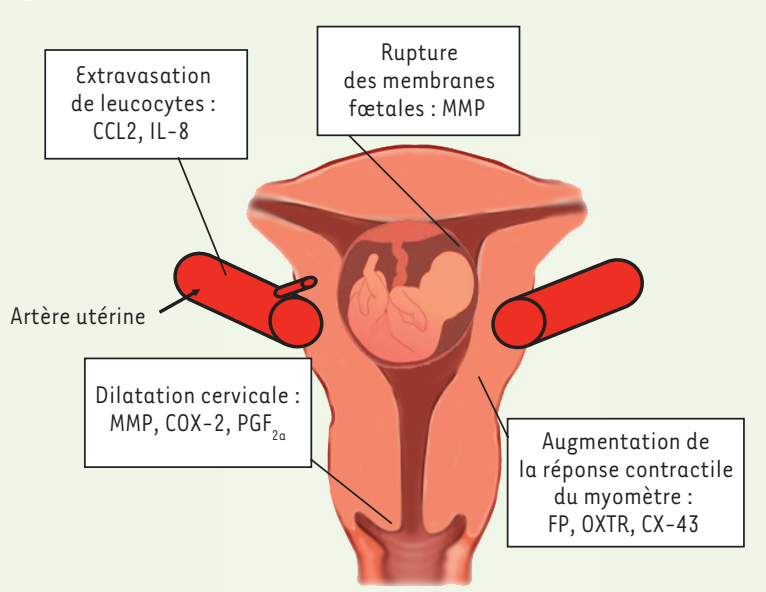

Figure 1. Cascade inflammatoire lors de l'activation utérine. A. La réaction inflammatoire provoque l'activation de l'utérus via les protéines d'activation de l'utérus (UAP) et mène ultimement au commencement du travail. B. Implication spécifique des UAP dans les changements physiologique associés au travail. OXTR : récepteur de l'ocytocine ; FP : récepteur de la prostaglandine $\mathrm{F}_{2 \alpha}$; MMP : métalloprotéinases matricielles. 
préventif. Les agents non compétitifs allostériques en développement seraient plus sélectifs et exposeraient donc à moins d'effets secondaires majeurs. L'utilisation d'un modulateur de récepteur(s) de cytokine (par exemple antiIL-IR ou anti-IL-6R) pour prévenir l'activation prématurée de l'utérus induite par l'inflammation pourrait potentiellement être indiqué chez les femmes asymptomatiques à risque $[9,10]$. $\diamond$

New insights to prevent preterm labor

\section{REMERCIEMENTS}

M.N.V. est financé par une bourse du Réseau de recherche en santé de la vision (RRSV) et par l'université de Montréal. J.P. est financée par le National council for scientific and technological development (CNPq) en collaboration avec le bureau canadien de l'éducation internationale $(C B \mid \varepsilon)$. S.C. détient une chaire canadienne de recherche translationnelle en vision et la chaire Leopoldine Wolfe en recherche translationnelle sur la dégénérescence maculaire liée à l'âge. Les projets en lien avec le travail préterme sont financés par les instituts de la recherche en santé du Canada (IRSC) conjointement avec la fondation Bill and Melinda Gates.

\section{LIENS D'INTÉRÊT}

Les auteurs déclarent n'avoir aucun lien d'intérêt concernant les données publiées dans cet article.

\section{RÉFÉRENCES}

1. Blencowe H, Cousens S, Chou D, et al. Born too soon: the global epidemiology of 15 million preterm births. Reprod Health 2013; 10 (suppl 1) : S2.

2. Bennett P. Preterm labour. In : Edmonds DK, ed. Dewhurst's textbook of obstetrics and gynaecology. UK : Wiley-Blackwell, 2012 : 338-55.

3. Olson DM, Christiaens I, Gracie S, et al. Emerging tocolytics: challenges in designing and testing drugs to delay preterm delivery and prolong pregnancy. Expert Opin Emerg Drugs 2008 ; 13 : 695-707.

4. Gomez-Lopez N, Tanaka S, Zaeem Z, et al. Maternal circulating leukocytes display early chemotactic responsiveness during late gestation. BMC Pregnancy Childbirth 2013; 13 (suppl 1) : S8.

5. Romero R, Espinoza J, Goncalves LF, et al.
Inflammation in preterm and term labour and delivery. Semin Fetal Neonatal Med 2006 ; 11 : 317-26.

6. Christiaens I, Zaragoza DB, Guilbert L, et al. Inflammatory processes in preterm and term parturition. J Reprod Immunol 2008 ; 79 : 50-7.

7. Keelan JA, Khan S, Yosaatmadja F, Mitchell MD. Prevention of inflammatory activation of human gestational membranes in an ex vivo model using a pharmacological NF-kappaB inhibitor. J Immunol $2009 ; 183: 5270-8$.

8. Kenakin T, Christopoulos A. Signalling bias in new drug discovery: detection, quantification and therapeutic impact. Nat Rev Drug Discov 2013 ; 12 : 205-16.

9. Goupil $\varepsilon$, Tassy D, Bourguet C, et al. A novel biased allosteric compound inhibitor of parturition selectively impedes the prostaglandin F2alphamediated Rho/ROCK signaling pathway. J Biol Chem $2010 ; 285: 25624-36$.

10. Böttcher B, Laterza RM, Wildt L, et al. A first-in-human study of PDC31 (prostaglandin F2alpha receptor inhibitor) in primary dysmenorrhea. Hum Reprod 2014 ; $29:$ 2465-73.

11. Jarreau PH. La prématurité. Med Sci (Paris) 2013 ; 29 : 819-20.

12. Lopez $\varepsilon$, Jarreau PH. Inflammation et dysplasie bronchopulmonaire. Med Sci (Paris) 2013 ; 29 : 823-5.

Société de philosophie des sciences (SPS)

Conférences Duhem 2015

Philosophie et médecine

Organisées en partenariat avec l'Académie nationale de médecine Mercredi 10 juin 2015 9h3o- 17h3o

Académie nationale de médecine

Salle des séances

16 rue Bonaparte - 75006 Paris

\section{Conférenciers invités}

Laurence Zitvogel (Inserm/Institut Gustave Roussy)

Immuno-oncologie ou la révolution thérapeutique en cancérologie

Joël Coste (Université Paris Descartes/EPHE)

Les maladies chroniques en médecine

Marina Cavazzana (Inserm/Université Paris-Descartes)

Gene therapy for hereditary disorders

Paul Thagard (University of Waterloo)

Explaining mental illness

Chaque conférence sera suivie d'un commentaire et d'une discussion

Programme détaillé : www.sps-philoscience.org

Contact : Thomas Pradeu, IHPST, 13 rue du Four, 75006 Paris

E-mail : thomas.pradeu@u-bordeaux.fr 